\title{
Experimental Vital Signs Estimation Using Commercially available IR-UWB Radar
}

\author{
Mounir Adjrad, Sandra Dudley and Mohammad Ghavami \\ Engineering and Design Department \\ London South Bank University \\ London, UK \\ Email: adjradm@1sbu.ac.uk, dudleyms@1sbu.ac.uk, ghavamim@1sbu.ac.uk
}

\begin{abstract}
This paper studies the feasibility of estimating vital signs exploiting commercially available Impulse Radio Ultra Wideband (IR-UWB) radar. The focus is on extracting breathing and heart beat rates following the consideration of a nonstationary analytical model for the reflected signal from the human body. The Hilbert-Huang Transform (HHT), which is adaptive to nonlinear and nonstationary signals, is proposed and applied to the intrinsic mode functions of the received signal providing frequency information evolving with time and quantifying the amount of variation due to different signal content contribution. Experimental results are presented demonstrating the effectiveness of the proposed technique for determining respiration and heartbeat rates.
\end{abstract}

\section{INTRODUCTION}

Impulse Radio Ultra Wideband (IR-UWB, referred to as UWB is the remainder of this paper) is a type of wireless technology that uses very short baseband (carrierless) pulses, typically in the order of nanoseconds [1]. Such wireless systems provide low system complexity, low cost and low power consumption. There are numerous advantages in exploiting UWB for biomedical applications because of its low radiation power, good coexistence with other wireless systems, and robustness to interference and multipath [1], [2]. A number of studies have investigated using UWB-based wireless sensing devices to detect vital signs for health care applications [3][6]. Microwave Doppler radar was proposed as non-invasive vital signs estimation technique [7]. However, Doppler radar techniques present two problems: 1) The difficulty in penetrating material and 2) the null point problem (occurring when the received signal and the radar local oscillator are either inphase or $180^{\circ}$ out of phase which would severely decrease the detection accuracy) [8]. Fourier analysis has become the most valuable tool in spectral data analysis and has consequently been applied to all kinds of data in many scientific or engineering disciplines; although it is strictly limited to linear systems and stationary data series [9]. Therefore, the Fourier spectrum can only be regarded as the coefficient function obtained by expanding a signal $x(t)$ into a family of an infinite number of waves generally in the form $\exp (i \omega t)$, which are completely unlocalised in time typifying the signal mathematically from global point of view. Thus, the Fourier spectrum essentially defines which spectral components, as well as their corresponding time-invariant amplitudes and phases, are embedded in the signal over the whole time span in which the signal was recorded.

In order to introduce a time-dependency in the Fourier transform technique, a simple and intuitive solution consists of pre-windowing the signal around a particular instant in time, calculating its Fourier transform, and repeating that procedure for each time step (with the assumption the signal being stationary in all windows). The resulting time-dependent spectrum (spectrogram) is called the short time Fourier transformation (STFT) and was first introduced by Gabor [10]. To localise an event precisely in time, the window width must be narrow, alternately the frequency resolution requires longer time spans. This leads to conflicting requirements (Heisenberg-Gabor inequality) and restrains this method from many practical applications, although it is still the most widely used technique in time-frequency analysis.

To avoid these restrictions, wavelet analysis, in continuous and discrete representation, was developed [11]. The central idea of the wavelet transform is to correlate a signal $x(t)$ with a family of zero-mean functions derived from an elementary function (mother wavelet), e.g. the complex-valued continuous Morlet mother wavelet derived from a plane wave modulated Gaussian envelope. In principle, the wavelet transform provides an amplitude spectrum of the signal $x(t)$ in time and frequency domain. But, even the wavelet analysis has certain limitations. Firstly, the chosen mother wavelet will significantly influence the result of the analysis, as the basic functions of wavelet transformation are fixed and do not necessarily match the shape of the considered data series in every time instant. Moreover, spectral wavelet analysis certainly underlies an uncertainty principle, indicating that a time or a frequency dependent information cannot be classified by the same accuracy, simultaneously. A high frequency component is precisely resolved in time domain, but at the same time inexact in frequency domain and vice versa for low frequency components.

A technique that overcomes the aforementioned limitations comprises exploiting the Hilbert-Huang Transformation (HHT) [12], [13]. The HHT decomposes any time-dependent signal into its individual embedded modes with the so-called Empirical Mode Decomposition (EMD). Applying the Hilbert Transformation (HT) to any of these disintegrated Intrinsic Mode Functions (IMF) subsequently generates distinct timedependent Hilbert amplitude or energy spectra. This implies, in all probability, that the HHT is capable of revealing entirely new physical insights for any nonlinear and non-stationary signal.

The paper is organised as follows. The problem formulation and modelisation along with the signal processing techniques 
used to estimate the respiration and heart rates are described in Section II. The proposed measurement setup and experimental results are presented and discussed in Section III. Concluding remarks are given in Section IV.

\section{Signal Model}

The aim of this paper is to estimate the breathing rate and heartbeat frequency. For this purpose, a mathematical model is developed so that the spectrum of the detected signal can be obtained and understood. This model is an extension of that proposed in [6] with the inclusion of heart motion. This permits a deep analysis of both harmonics and cross products (intermodulation). The sections below demonstrate the importance of making this complete analysis, since the large amplitude of breath harmonics and frequently that of cross products make heart rate measurement a challenge, especially when they are close to the heart frequency range. When the transmitted pulse hits the human target, part of it is reflected due to the high reflectivity of the human body [3], [4]. The time-of-arrival (ToA) of this pulse is denoted by $\tau_{0}$ and depends on the antenna-to-target distance, $d_{0}$. Due to respiration and heart motion, the chest cavity expands and contracts periodically, so the distance traveled, $d(t)$, varies periodically around the nominal distance $d_{0}$. For vital signs monitoring, the body movement caused by both respiration and heartbeat must be detected:

$$
d(t)=d_{0}+m(t)=d_{0}+m_{b} \sin \left(2 \pi f_{b} t\right)+m_{h} \sin \left(2 \pi f_{h} t\right)
$$

where $m_{b}$ and $m_{h}$ are the respiration and heartbeat displacement amplitudes, $f_{b}$ and $f_{h}$ are the respiration and heartbeat frequencies, respectively. In this situation, the received signal can be represented as the sum of the responses of the channel, and the variation due to the respiration and heartbeat:

$$
r(t, \tau)=\sum_{i} A_{i} p\left(\tau-\tau_{i}\right)+A p\left(\tau-\tau_{d}(t)\right)
$$

where $p(t)$ is the normalised received pulse, $A_{i}$ is the amplitude of each multipath component, $\tau_{i}$ its delay, and $A$ is the amplitude of the pulse reflected from the human body. From (2) it is evident that respiration and heart movements modulate the received signal. The time delay $\tau_{d}$ associated with the vital sign is modeled as the sum of TOA $\tau_{0}$ plus two sinusoidal delays associated to respiration and heartbeat displacements:

$$
\tau_{d}(t)=2 d(t) / c=\tau_{0}+\tau_{b} \sin \left(2 \pi f_{b} t\right)+\tau_{h} \sin \left(2 \pi f_{h} t\right)
$$

where $c$ is the light velocity, and $\tau_{b}$ and $\tau_{h}$ are the respiration and heartbeat displacements, respectively. The received waveforms are measured at discrete instants in slow time $t=n T_{s}$ with $n=1,2, \ldots, N . N$ discrete-time sequences are stored after the received signal is sampled and these values are stored in a matrix $R$, the elements of which are:

$$
R[n, m]=r\left(\tau=n T_{f}, t=m T_{s}\right)
$$

where $T_{f}$ is the sampling period in fast-time. In a static environment, the resulting clutter can be considered as a DCcomponent in the slow-time direction. Consequently, the only movement is caused by the person's respiration and heart activity, from (2) it is clear that background clutter does not depend on slow-time $t$. Thus, the background clutter can be removed by filtering the signal. This can be performed by subtracting the average of all received waveforms from the original signal (a new matrix $X$ will be obtained by subtracting the average of all the rows in $R$ from each row).

$$
\begin{aligned}
x(t, \tau) & =r(t, \tau)-\lim _{T \rightarrow \infty} \frac{1}{T} \int_{0}^{T} r(t, \tau) d t \\
& =A p\left(\tau-\tau_{d}(t)\right)-r_{0}(\tau)
\end{aligned}
$$

The DC component $r_{0}(\tau)$ is canceled by subtracting the average of all samples in fast-time (the result is saved in a new matrix $Y$ that is obtained by subtracting the average of all columns in $X$ from each column). The signal of interest is:

$$
y(t, \tau)=x(t, \tau)-x_{0}(\tau)=A p\left(\tau-\tau_{d}(t)\right)
$$

The goal is to obtain the breathing frequency $f_{b}$ and heart rate $f_{h}$. To this end, the concept of empirical mode decomposition (EMD) and the Hilbert spectrum (HS) is applied to further process the resulting $y$ matrix.

The key feature of EMD is to decompose a signal into so-called intrinsic mode functions (IMFs). Furthermore, the Hilbert spectral analysis of intrinsic mode functions provides frequency information evolving with time and quantifies the amount of variation due to oscillation at different time scales and time locations. The essential step extracting an IMF is to identify an oscillation embedded in a signal from local time scale. A component for a given time scale can be regarded as the composition of repeated intrinsic oscillation which is symmetric to its local mean, zero. Thus the first step to define intrinsic oscillation is to detect local extrema or zero-crossings. Once local extrema is obtained, the intrinsic mode function is derived through a procedure called sifting procedure.

Huang et al [12] suggested a data-adapted algorithm extracting a sinusoidal wave or equivalently a frequency from a given signal. First, identify the local extrema and generate two functions namely the upper envelope and lower envelope by interpolating local maxima and local minima, respectively. Second, take their average, which will produce a lower frequency component than the original signal. Third, by subtracting the envelope mean from the original signal, the highly oscillated pattern is separated. Huang et al defined an oscillating wave as an intrinsic mode function if it satisfies two conditions: (1) the number of extrema and the number of zerocrossings differs only by one and (2) the local average is zero. If the conditions of IMF are not satisfied after one iteration of aforementioned procedure, the same procedure is applied to the residue signal until properties of IMF are satisfied. This iterative process is called sifting. Once the highest frequency is removed from a signal, the same procedure is applied to the residue signal to identify next highest frequency. The residue is considered a new signal to decompose and the procedure is repeated on this latter.

When a signal is subject to non-stationarity so that the frequency and amplitude change over time, it is necessary to have a more flexible and extended notion of frequency. The concept of instantaneous frequency is then exploited through the use of the Hilbert transform (For a comprehensive explanation of the Hilbert transform, refer to Cohen [14]). Following decomposing a signal into IMFs with EMD thereby preserving any local property in the time domain, we can 


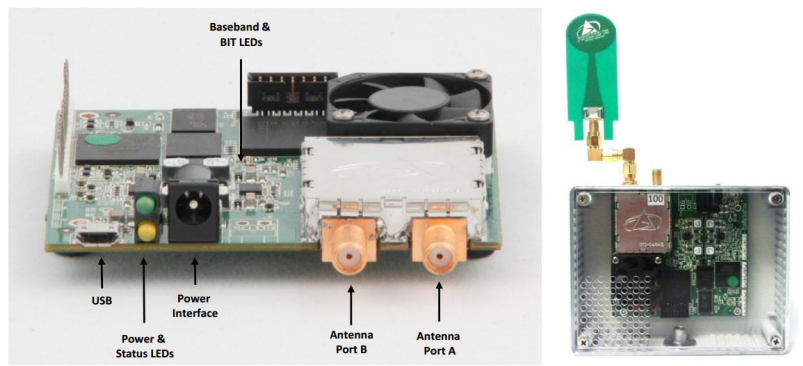

Fig. 1. PulsON P410 UWB tranceiver.
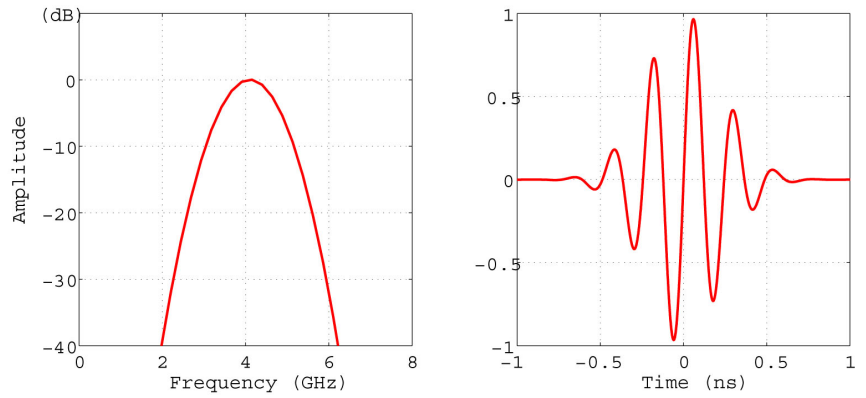

Fig. 2. Transmitted waveform in frequency (Left) and time (Right).

extract localised information in the frequency domain with the Hilbert transform and identify hidden local structures embedded in the original signal. The local information can be described by the Hilbert spectrum which is amplitude and instantaneous frequency representation with respect to time.

\section{Measurement Setup AND ExPerimental RESULTS}

The UWB radar system comprises two Broadspec antennas and one PulsON P410 transceiver provided by TimeDomain [15] (see Figure 1). Pulses are transmitted via one antenna and the target reflected signal is received by the second antenna. The raw data samples are then sent to a host PC for processing. TimeDomain exploits coherent signal processing with low duty cycle transmissions and pulse repetition rates of $10 \mathrm{MHz}$. The UWB transmissions typically consist of a packet of between several thousand and a few hundred thousand coherently transmitted pulses. The signal energy can be spread over multiple pulses thanks to coherent mode of transmission, hence providing an increase in the energy per bit and thereby the signal to noise ratio (SNR) [15]. The waveform generated by the transceiver is a Gaussian modulated sinusoidal pulse with the following analytical model:

$$
s(t)=\exp \left[-t^{2} / 2 \sigma^{2}\right] \sin \left(2 \pi f_{c} t\right)
$$

It is centred at $f_{c}=4.3 \mathrm{GHz}$, occupies more than $2 \mathrm{GHz}$ of bandwidth and achieves an effective RF bandwidth of 1.4 $\mathrm{GHz}$ (Figure. 2).

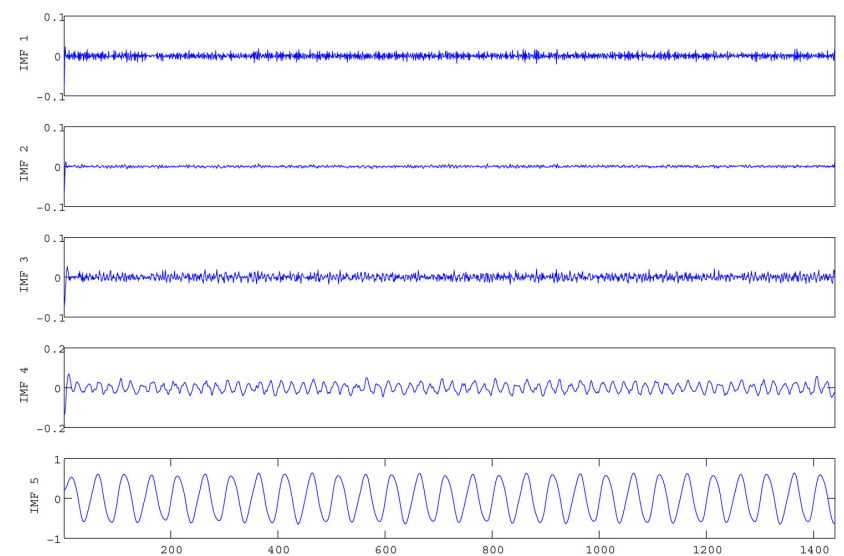

Fig. 3. IMFs 1-5.

The antennas have the following characteristics [14]: very good time response where they can transmit and receive UWB pulses effectively with minimum distortion, little ringing and small reflection, compact size and low profile, omnidirectional radiation beam and good reflection coefficient. Note that it is a requirement that the target should be a certain distance away from the antennas for this radar system to have accurate measurements, because then the human body reflected pulse can be distinguished clearly from the direct coupling pulse between the antennas. Since the pulse used in this work has a width of about 1 ns (Figure 2), the distance between the target and the antennas should be larger than $300 \mathrm{~mm}$. It is then set in this work that this minimum distance should be $750 \mathrm{~mm}$.

In the experimental results, 1024 waveforms are acquired, sampled and averaged to improve the signal-to-noise ratio. The recorded duration is $30 \mathrm{~s}$. The actual heart beat rate was 1.22 $\mathrm{Hz}$ corresponding to 73 beats per minute, while the breathing rate was $0.42 \mathrm{~Hz}$ corresponding to 25 breaths per minute (Typically, in relaxed human beings the heart can cause chest displacements of $0.08 \mathrm{~mm}$, and respiration displacements of between $0.1 \mathrm{~mm}$ and several millimeters, depending on the person [16]).

Figure 3 illustrates the IMFs up to the fifth one where by observing IMF4 and IMF5 one can identify the separate contributions of the heart beat and respiratory rates, respectively.

Figure 4 shows the Hilbert energy spectrum following the extraction of the IMFs of the received UWB signal, and illustrating an estimation of the heart beat and respiratory rate (with actual frequencies of $1.22 \mathrm{~Hz}$ and $0.42 \mathrm{~Hz}$, respectively).

It is important to clarify that within the experimental setup the measurements were taken on a subject instructed to remain static during the measurement period. Approaches for cancelation of unwanted motion, as a pre-processing step of the raw data, will be investigated in future work to make this approach applicable to more practical scenarios. Similarly, future effort will consider the case of simultaneously monitoring multiple subjects. 


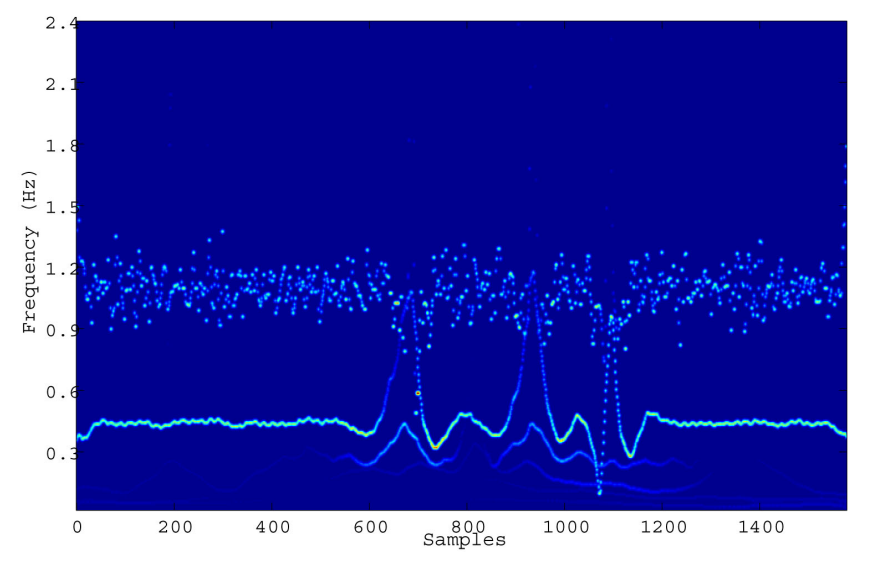

Fig. 4. Hilbert energy spectrum.

\section{CONClusion}

Breathing and heart beat rate estimation were successfully performed exploiting commercially available UWB radar operating within the FCC mask. IMFs through EMD offered a multi-resolution tool and spectral analysis provided local information with time-varying amplitude and phase according to actual component scales. the HHT is proving a powerful tool to analyzing nonlinear and non-stationary vital sign signals in the time-frequency plane and providing a more physically meaningful interpretation.

\section{ACKNOWLEDGMENT}

The work is sponsored by the national EPSRC DANCER project (EP/K002473/1; EP/K002643/1).

\section{REFERENCES}

[1] M. Ghavami, L. Michael, and R. Kohno. Ultra Wideband Signals and Systems in Communication Engineering. Wiley \& Sons, 2007.

[2] Zhuang, W., X. Shen, and Q. Bi, "Ultra-wideband wireless communications," Wirel. Commun. Mob. Comput., No. 3, 2003.

[3] E. M. Staderini, "UWB radars in medicine," IEEE Aerospace and Electronic Systems Magazine, No. 1, 2002.

[4] C. G. Bilich,"Bio-medical sensing using ultra wideband communications and radar technology : A feasibility study," IEEE Pervasive Health Conference and Workshops, Nov. 2006.

[5] Ossberger, G., T. Buchegger, E. Schimback, A. Stelzer, and R. Weigel, "Non-invasive respiratory movement detection and monitoring of hidden humans using ultra wideband pulse radar," Proc. of the 2004 International Workshop on Ultra Wideband Systems Joint with Conference on Ultra Wideband Systems and Technologies, Piscataway, NJ, USA, 2004.

[6] Venkatesh, S., C. Anderson, N. V. Rivera, and R. M. Buehrer, "Implementation and analysis of respiration-rate estimation using impulsebased UWB," 2005 IEEE Military Communications Conference (IEEE Milcom'05), Vol. 5, Oct. 2005.

[7] Ivashov, S. I., V. V. Razevig, A. P. Sheyko, and I. A. Vasilyev, "Detection of human breathing and heartbeat by remote radar," Progress In Electromagnetic Research Symposium, Pisa, Italy, Mar. 2004.

[8] Lubecke, O. B., P. W. Ong, and V. M. Lubecke, "10 GHz doppler radar sensing of respiration and heart movement," 2002 IEEE Proceedings of the IEEE 28th Annual Northeast Bioengineering Conference, Apr. 2002.

[9] Titchmarsh, E. C., "Introduction to the theory of Fourier integrals, Ox. U. Pr.1948.

[10] Gabor, D., "Theory of Communication, IEE J. Comm. Eng. 1993.
[11] Daubechies, I.,"Ten Lectures on Wavelets, Soc. f. Ind. \& App. M. 1992.

[12] Huang, N.E., Shen, Z., Long, S., Wu, M.C., Shih, H.H., Zheng, Q., Yen, N.-C., Tung, C. C., Liu, H. H., "The Empirical Mode Decomposition and Hilbert Spectrum for nonlinear and non-stationary time series analysis, Proc. R. Soc. London A, 454, 1998.

[13] Huang, N. E., Shen, Z., Long, S., "A new view of nonlinear water waves: The Hilbert Spectrum, Annual Review of Fluid Mechanics, 1999.

[14] L. Cohen. Time-Frequency Analysis. Prentice-Hall, 1995.

[15] TimeDomain, www.timedomain.com.

[16] Singh, M. and G. Ramachandran, "Reconstruction of sequential cardiac in-plane displacement patterns on the chest wall by laser speckle interferometry," IEEE Trans. Biomed. Eng., Vol. 38, No. 5, 1991. 